\title{
SEM and Auger Electron Spectroscopy of Liquid Water through Graphene Membrane
}

\author{
Hongxuan Guo ${ }^{1,2}$, Alexander Yulaev ${ }^{1,2,3}$, Evgheni Strelcov ${ }^{1,2}$, and Andrei Kolmakov ${ }^{1,2}$ \\ 1. Center for Nanoscale Science and Technology, NIST, Gaithersburg, MD 20899, USA \\ 2. Maryland Nano Center, University of Maryland, College Park, MD 20742, USA \\ 3. Department of Materials Science and Engineering, University of Maryland, College Park, MD \\ 20742, USA.
}

Scanning electron microscopy (SEM) and Auger spectroscopy (AES) are important techniques to characterize the surface morphology and composition of materials. However, their applications for observation and characterization of liquids or aqueous samples is limited due to very short mean free path of low energy electrons in a condense phase or dense gases. Therefore, such experiments are currently conducted at synchrotron radiation facilities using sophisticated differentially pumped instrumentation [1, 2, 3]. Few years ago we proposed to use 2D materials as electron transparent membranes which separate high vacuum environment of the electron spectrometer from a liquid sample $[4,5]$. Recently, we developed a new graphene liquid cell platform based on multichannel capillary array (MCA) [6]. An electrochemical version of the graphene liquid cell has been fabricated via coating the back side of the MCA with Pt counter electrode using atomic layer deposition (ALD).

In this presentation, we report on SEM and AES characterization of liquid water inside microchannels capped by double-layer graphene (Fig. 1a). Figure 1b shows $\mathrm{O}_{\text {KLl }}$ and $\mathrm{C}_{\mathrm{KLL}}$ Auger spectra from water filled and empty channels measured through the graphene membrane. Comparing the sets of these differential AES spectra, we were able to estimate the effective thickness of the graphene membrane as well as probing depth in liquid water (Fig.1c). While AES probes very few water layers beneath graphene, energy dispersive spectroscopy (EDS) provides the measureable spectra (Fig. 2a) and the corresponding chemical maps (Fig. 2b), up to two microns deep in to the water. As an example of in situ electrochemical studies, Fig. 3 demonstrates $\mathrm{Cu}$ electrodeposition on graphene working electrode from $\mathrm{CuSO}_{4}$ aqueous solution. During anodic polarization, $\mathrm{Cu}$ nano-crystals grow on the back side of the graphene, while at the reverse bias they were stripped away. In summary, we developed a new environmental sample platform for scanning electron microscopy and spectroscopy measurements in liquids and gases. The platform is based on a multichannel array capped with a bilayer graphene which can be filled with a wide variety of liquids. The applicability of SEM, AES, EDS have been demonstrated using pure water and a model electrochemical reaction. The cell enables the characterization of materials relevant to energy, catalysis and biomedical applications [7].

References:

[1]. B. Winter, M. Faubel, Chem. Rev., 106 (2006), 1176-1211.

[2]. G. Olivieri, et al, J. Electron. Spectrosc. Relat. Phenom., 216(2017), 1-16.

[3]. P. Slavíček et al, J. Phys. Chem. Lett., 7(2016), 234-243

[4]. A. Kolmakov, et al, Nat. Nanotechnol., 6 (2011), 651-657

[5]. J. D. Stoll, A. Kolmakov, Nanotechnology, 23 (2012), 505704.

[6]. H. Guo, et al, Nano Lett. 17(2017), 1034-1041. 
[7]. The authors acknowledge funding from the Cooperative Research Agreement between the University of Maryland and the National Institute of Standards and Technology Center for Nanoscale Science and Technology, Award 70NANB10H193, through the University of Maryland.

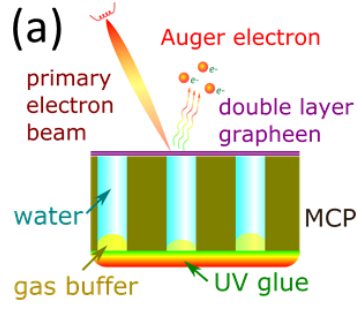

(c)

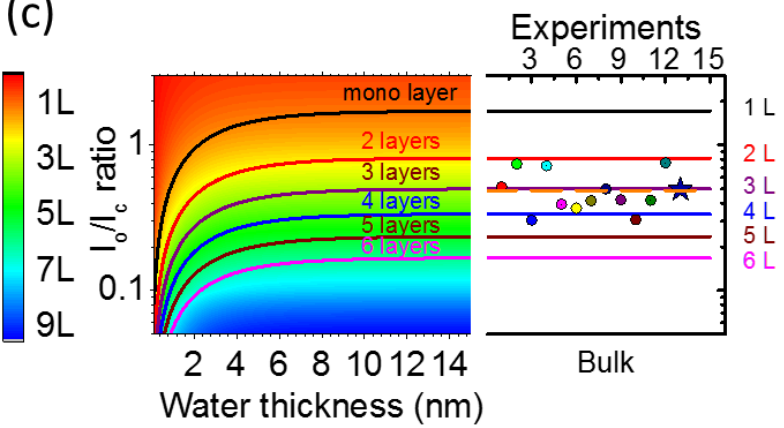

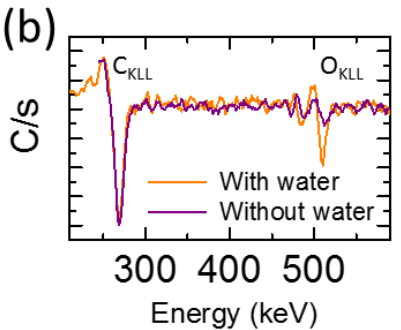

Figure 1. Auger spectra of water under double layer graphene
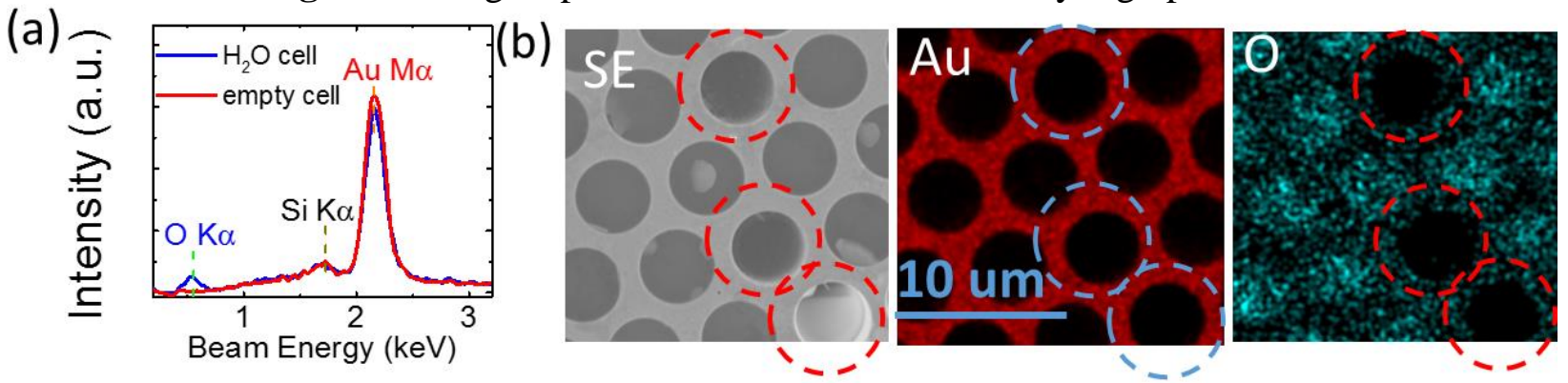

Figure 2. EDS characterization of water in micro-channels

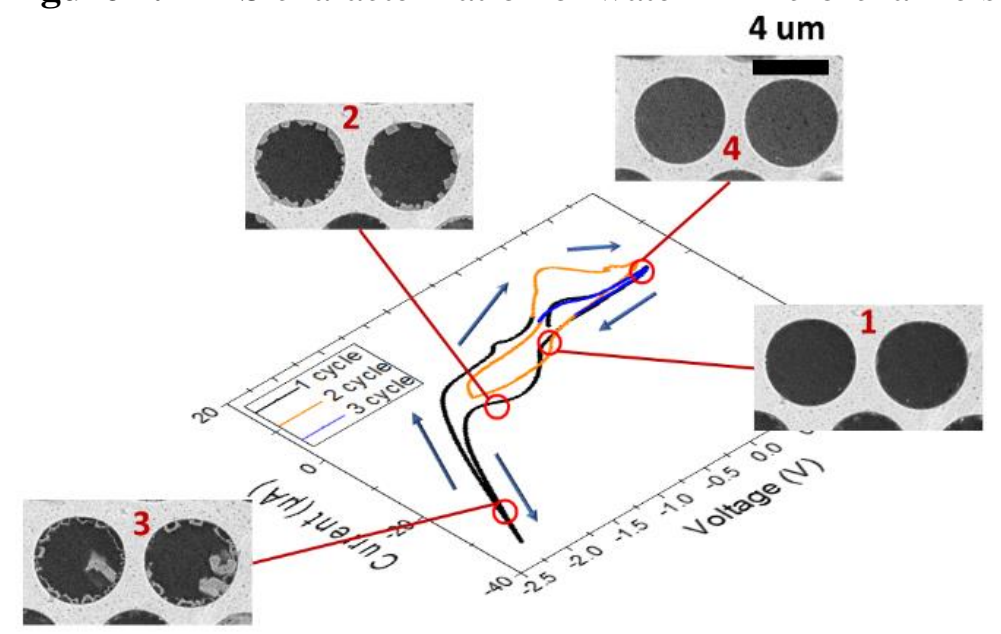

Figure 3. In-situ observation of electrochemical reaction in aqueous solution. 